\title{
ANALYSIS OF SPECTRAL APPROXIMATIONS USING PROLATE SPHEROIDAL WAVE FUNCTIONS
}

\author{
LI-LIAN WANG
}

\begin{abstract}
In this paper, the approximation properties of the prolate spheroidal wave functions of order zero (PSWFs) are studied, and a set of optimal error estimates are derived for the PSWF approximation of non-periodic functions in Sobolev spaces. These results serve as an indispensable tool for the analysis of PSWF spectral methods. A PSWF spectral-Galerkin method is proposed and analyzed for elliptic-type equations. Illustrative numerical results consistent with the theoretical analysis are also presented.
\end{abstract}

\section{INTRODUCTION}

The prolate spheroidal wave functions, originated from the context of separation of variables for the Helmholtz equation in spheroidal coordinates (see, e.g., [20, 12]), have been extensively used for a variety of physical and engineering applications, such as wave scattering, signal processing, and antenna theory (see, for instance, 3 , 11, 17]). Most notably, a series of papers by Slepian et al. [25, 19, 26] and the recent works by Xiao and Rokhlin et al. [33, 32, 24, 22, have shown that the PSWFs are a natural and optimal apparatus for approximating bandlimited functions. Recently, there has been a growing interest in developing numerical methods using PSWFs as basis functions, which include in particular the PSWF wavelets [31, 30, 29] and the PSWF spectral/spectral-element methods [7, 8, 3, 10, 18, 17, 27.

Boyd [7] and Chen et al. [10, have demonstrated that spectral accuracy can be achieved when the PSWFs (with a suitable choice of the bandwidth parameter) are used to approximate non-periodic smooth functions on finite intervals, and the PSWF spectral methods enjoy modest advantages over their polynomial-based counterparts: (i) enable fewer points per wavelength to resolve waves; (ii) use quasiuniformly distributed collocation points allowing for a larger time step in explicit time-marching schemes, and (iii) achieve a better resolution near the center of the computational domain. Moreover, Boyd [8, 7] showed that, by a straightforward basis and differentiation matrix swamping, the prolate element method, built on quasi-uniform meshes, promises to outperform the conventional Legendre spectral

Received by the editor July 16, 2008 and, in revised form, December 30, 2008.

2000 Mathematics Subject Classification. Primary 65N35, 65N22, 65F05, 35J05.

Key words and phrases. Prolate spheroidal wave functions, bandlimited functions, approximations in Sobolev spaces, spectral methods, quasi-uniform grids.

This work is partially supported by AcRF Tier 1 Grant RG58/08, Singapore MOE Grant T207B2202, and Singapore NRF2007IDM-IDM002-010.

(C)2009 American Mathematical Society Reverts to public domain 28 years from publication 
element method. In addition, Moore et al. 21 addressed that the PSWF series is potentially optimal over more conventional orthogonal expansions for discontinuous functions such as the square wave, among others. More interestingly, Kovvali et al. [18. proposed a fast algorithm for performing matrix-vector multiplication involving prolate spectral differentiation and interpolation with an $O(N)$ complexity.

Although there is an increasing number of papers devoted to analytic properties and numerical evaluation of the PSWFs (see, e.g., 33, 5, 6, 21, 22, 13, 34 and the references therein), the approximability of the PSWFs is studied in a limited number of papers. Some error analysis for approximation of bandlimited functions by PSWFs was carried out in the note 24] (also see Xiao's thesis [32]). The first result on error estimates of the PSWF series expansions for non-periodic functions in Sobolev spaces was derived in Chen et al. 10] and Boyd [7] (refer to Theorem 3.2 below), which explicitly indicates the feasible choice of the bandwidth parameter for achieving a spectral accuracy. However, the order of convergence is somehow suboptimal, in particular for fixed bandwidth parameters, and the underlying analysis, which is essentially based on a delicate analysis of the decay properties of the coefficients in Legendre expansions of the PSWFs, is non-trivial to extend to estimates in higher-order Sobolev spaces. In this paper, we take a different approach, which leads to a set of optimal approximation results with a more concise analysis. These results serve as a main ingredient for the analysis of PSWF spectral methods. We also introduce a PSWF spectral-Galerkin method using a modal basis consisting of compact linear combinations of integration of PSWFs, which results in an effective PSWF Galerkin approximation to PDEs, and therefore provides a viable alternative to the PSWF collocation/pseudospectral method.

The rest of the paper is organized as follows. In the next section, we collect and extend some properties of the PSWFs to be used throughout the paper. In Section 3 , we show that a super-geometric convergence can be attained when the PSWFs are used to approximate bandlimited functions. More importantly, we derive a set of PSWF approximation results in Sobolev spaces. In Section 4, we analyze and implement a PSWF spectral-Galerkin method for some model PDEs, and then provide some numerical results to confirm and support the theoretical analysis. The final section is for some concluding remarks and discussions.

We now introduce some notation to be used throughout the paper. Let $I:=$ $(-1,1)$ and let $\varpi$ be a generic weight function defined in $I$. The weighted Sobolev spaces $H_{\varpi}^{s}(I)(s=0,1,2, \ldots)$ can be defined as usual with inner products, norms and semi-norms denoted by $(\cdot, \cdot)_{s, \varpi},\|\cdot\|_{s, \varpi}$ and $|\cdot|_{s, \varpi}$, respectively. For real $s>0, H_{\varpi}^{s}(I)$ is defined by space interpolation as in [1. In particular, we have $L_{\varpi}^{2}(I)=H_{\varpi}^{0}(I)$, and we use $(\cdot, \cdot)_{\varpi}$ and $\|\cdot\|_{\varpi}$ to denote the $\varpi$-weighted $L^{2}$-inner produce and norm, respectively. The subscript $\varpi$ will be omitted from the notation in cases of $\varpi \equiv 1$.

We will use $\partial_{x}^{k}$ to denote the ordinary derivative $\frac{d^{k}}{d x^{k}}$, whenever no confusion may arise. We denote by $C$ a generic positive constant independent of any function, bandwidth parameter and discretization parameters. We use the expression $A \lesssim B$ to mean that there exists a generic positive constant $C$ such that $A \leq C B$.

\section{Prolate spheroidal Wave FunCtions}

In this section, we review and extend some relevant properties of the PSWFs, most of which can be founded in, e.g., [20, 12, 25, 19, 26, 33]. 
For any real number $c \geq 0$, the PSWFs of degree $n$, denoted by $\psi_{n}^{c}(x)$, are the eigenfunctions of the Sturm-Liouville equation

$$
\partial_{x}\left(\left(1-x^{2}\right) \partial_{x} \psi_{n}^{c}\right)+\left(\chi_{n}^{c}-c^{2} x^{2}\right) \psi_{n}^{c}=0, \quad x \in I:=(-1,1),
$$

with the corresponding eigenvalues $\chi_{n}^{c}$. Hence, the constant $c$ is called the bandwidth parameter. In particular, if $c=0$, the PSWFs are reduced to the classical Legendre polynomials with the associated eigenvalues $\chi_{n}^{0}=n(n+1)$. The PSWFs can be viewed as a generalization of the Legendre polynomials, but oscillate more uniformly as $c$ increases (cf. Boyd [7). Indeed, the PSWFs share the following important properties with the Legendre polynomials.

Lemma 2.1. For any $c>0$,

(i) $\left\{\psi_{n}^{c}(x)\right\}_{n=0}^{\infty}$ are all real, smooth and form a complete orthonormal system in $L^{2}(I)$, namely,

$$
\int_{-1}^{1} \psi_{n}^{c}(x) \psi_{m}^{c}(x) d x=\delta_{m n},
$$

where $\delta_{m n}$ is the Kronecker symbol.

(ii) $\left\{\chi_{n}^{c}\right\}_{n=0}^{\infty}$ are all real, positive, simple and ordered as

$$
0<\chi_{0}^{c}<\chi_{1}^{c}<\cdots<\chi_{n}^{c}<\cdots .
$$

(iii) $\left\{\psi_{n}^{c}(x)\right\}_{n=0}^{\infty}$ with even $n$ are even functions of $x$, and those with odd $n$ are odd functions.

(iv) $\psi_{n}^{c}(x)$ has exactly $n$ real distinct roots in the interval $(-1,1)$.

Next, we have from Theorem 12 of Rokhlin and Xiao 22] that for any $c>0$,

$$
\left|\psi_{n}^{c}(1)\right|<\sqrt{n+\frac{1}{2}}, \quad \forall n \geq 0,
$$

and by Formula (11) of Shkolnisky et al. 24,

$$
\max _{0 \leq j \leq n} \max _{|x| \leq 1}\left|\psi_{j}^{c}(x)\right| \leq 2 \sqrt{n}, \quad \forall n \geq 1, \quad \forall c>0 .
$$

We now examine the behaviors of the eigenvalues $\left\{\chi_{n}^{c}\right\}$. The following estimate is found useful in the sequel (see Appendix $\mathrm{A}$ for the proof).

Lemma 2.2. For any $c>0$,

$$
n(n+1)<\chi_{n}^{c}<n(n+1)+c^{2}, \quad \forall n \geq 0 .
$$

For large $n$, we deduce from Formula (64) of Rokhlin and Xiao 22] that

$$
\chi_{n}^{c}=n(n+1)+\frac{c^{2}}{2}+\frac{c^{2}\left(4+c^{2}\right)}{32 n^{2}}\left(1-\frac{1}{n}+O\left(n^{-2}\right)\right), \quad \forall n \gg 1 .
$$

As a numerical illustration, we plot in Figure 1 the error $\log _{10}\left(\left|\chi_{n}^{c}-\tilde{\chi}_{n}^{c}\right|\right)$ with $\tilde{\chi}_{n}^{c}:=n(n+1)+\frac{c^{2}}{2}+\frac{c^{2}\left(4+c^{2}\right)}{32 n^{2}}\left(1-n^{-1}\right)$, for various $n$ and $c$ (see the caption). It shows that $\tilde{\chi}_{n}^{c}$ provides a fairly reasonable approximation to $\chi_{n}^{c}$ for $n>c$.

For large $c$, the eigenvalues behave like (see, e.g., Formula (18) of Boyd [7])

$$
\chi_{n}^{c}=c(2 n+1)-\frac{n^{2}+n+\frac{3}{2}}{2}+O\left(c^{-1}\right), \quad \forall c \gg 1 .
$$



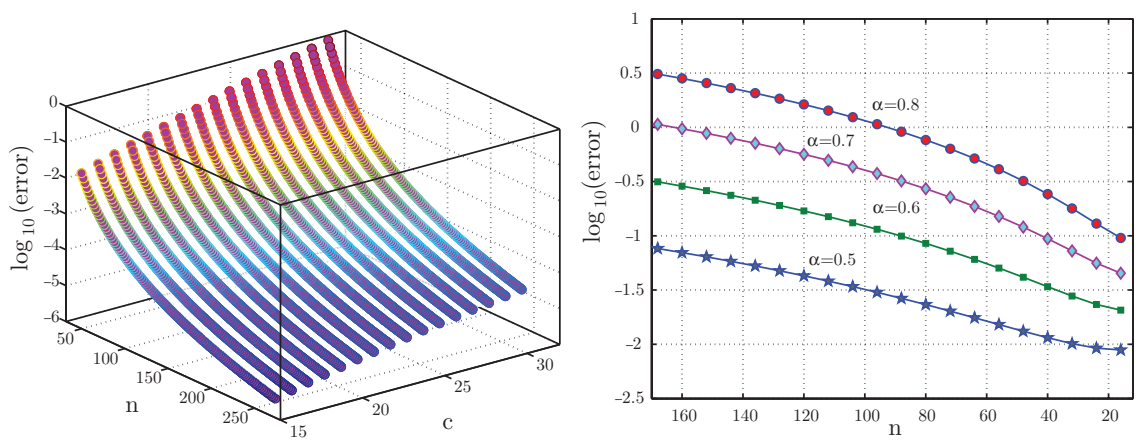

FiguRE 1. $\log _{10}\left(\left|\chi_{n}^{c}-\tilde{\chi}_{n}^{c}\right|\right)$ for various $n \in[32,256]$ and $c \in$ $[16,32]$ (left), and for $c=\alpha n$ with various $n \in[16,168]$ and $\alpha=$ $0.5,0.6,0.7,0.8$ (right).

It is remarkable that the PSWFs are also the eigenfunctions of the compact integral operator $F_{c}: L^{2}(I) \rightarrow L^{2}(I)$, defined by

$$
F_{c}[\phi](x)=\int_{-1}^{1} e^{\mathrm{i} c x t} \phi(t) d t, \quad \forall x \in(-1,1), \quad \forall c>0 ;
$$

namely,

$$
\mathrm{i}^{n} \lambda_{n}^{c} \psi_{n}^{c}(x)=\int_{-1}^{1} e^{\mathrm{i} c x t} \psi_{n}^{c}(t) d t, \quad \forall x \in(-1,1) .
$$

The corresponding eigenvalues $\left\{\lambda_{n}^{c}\right\}$ (modulo the factor $\mathrm{i}^{n}$ ) are all real, positive, simple and can be ordered as 1

$$
\lambda_{0}^{c}>\lambda_{1}^{c}>\cdots>\lambda_{n}^{c}>\cdots>0, \quad \forall c>0 .
$$

One verifies readily that the PSWFs satisfy

$$
\mu_{n}^{c} \psi_{n}^{c}(x)=\int_{-1}^{1} \frac{\sin (c(x-t))}{x-t} \psi_{n}^{c}(t) d t:=Q_{c}\left[\psi_{n}^{c}\right](x) \quad \text { with } \mu_{n}^{c}=\frac{c}{2}\left|\lambda_{n}^{c}\right|^{2},
$$

and one also notices that $Q_{c}=\frac{c}{2} F_{c}^{*} \circ F_{c}$.

It is worthwhile to have a quantitative study of the eigenvalues $\left\{\lambda_{n}^{c}\right\}$ and $\left\{\mu_{n}^{c}\right\}$. An explicit expression for $\lambda_{n}^{c}$ in terms of $\psi_{n}^{c}(1)$ is given by Theorem 9 of Rokhlin and Xiao 22 :

$$
\lambda_{n}^{c}=\frac{\sqrt{\pi} c^{n}(n !)^{2}}{(2 n) ! \Gamma(n+3 / 2)} \cdot \exp \left(\int_{0}^{c}\left(\frac{2\left(\psi_{n}^{\tau}(1)\right)^{2}-1}{2 \tau}-\frac{n}{\tau}\right) d \tau\right), \quad \forall c>0 .
$$

We deduce from (2.4) that the value of the integral within the exponential is negative, which implies that

$$
\lambda_{n}^{c}<\frac{\sqrt{\pi} c^{n}(n !)^{2}}{(2 n) ! \Gamma(n+3 / 2)}:=\nu_{n}^{c}, \quad \forall c>0 .
$$

\footnotetext{
${ }^{1}$ Notice that the eigenvalues $\chi_{n}^{c}$ and $\lambda_{n}^{c}$ belong to the same PSWF $\psi_{n}^{c}(x)$, and the coexistence of the ordering (2.3) and (2.11) was proved in Slepian and Pollak 25.
} 
We next show that $\lambda_{n}^{c}$ decays exponentially with respect to $n$, and we illustrate that the upper bound $\nu_{n}^{c}$ provides a good approximation to $\lambda_{n}^{c}$ for modest large $n$. Indeed, by Stirling's formula:

$$
\Gamma(x)=\sqrt{2 \pi} x^{x-1 / 2} e^{-x}\left\{1+\frac{1}{12 x}+O\left(x^{-3}\right)\right\}, \quad x \gg 1,
$$

and the identity $\Gamma(n+1)=n$ !, we have that for $n \gg 1$,

$$
\lambda_{n}^{c} \sim \frac{1}{e} \sqrt{\frac{\pi}{2}}\left(\frac{e c}{4}\right)^{n}\left(n+\frac{1}{2}\right)^{-n-\frac{1}{2}}=\frac{1}{e} \sqrt{\frac{2 \pi}{e c}}\left(\frac{4}{e c}\left(n+\frac{1}{2}\right)\right)^{-n-\frac{1}{2}} .
$$

Therefore, $\lambda_{n}^{c}$ decays super-geometrically as $n$ grows; namely,

$$
\lambda_{n}^{c} \sim \exp (\tilde{n}(\kappa-\log \tilde{n})), \quad \kappa=\log \frac{e c}{4}, \quad \tilde{n}=n+\frac{1}{2} \gg 1 .
$$

The above result confirms in particular Conjecture 1 in Boyd [5. We also notice that for all $c>0, \sum_{n=0}^{\infty}\left(\lambda_{n}^{c}\right)^{2}=4$ (see Formula (143) of [33]).

To visualize the behavior of the eigenvalues $\left\{\lambda_{n}^{c}\right\}$, we plot in Figure 2 (left) the profiles of $\log _{10}\left(\lambda_{n}^{c}\right)$ with various $n \in(0,100]$ and $c \in(0,80]$, computed by the formulas (2.21) -(2.22) below. We see that the eigenvalues begin to decay (super-) geometrically, when $n>\frac{e c}{4}-\frac{1}{2}$. On the right of Figure 2, we depict the graphs of $\log _{10}\left(\lambda_{n}^{c}\right)$ against $\log _{10}\left(\nu_{n}^{c}\right)$ for $n \geq \frac{e c}{4}-\frac{1}{2}$ with $c=20,30, \ldots, 90$ (from bottom to top), where the vertical dotted lines indicate the position of the smallest integer greater than $\frac{e c}{4}-\frac{1}{2}$.
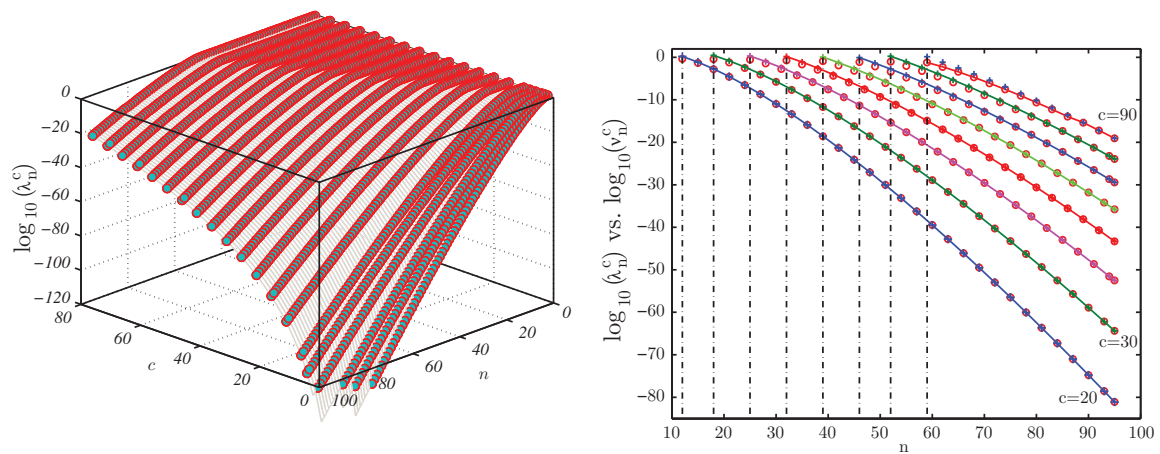

Figure 2. Profiles of $\log _{10}\left(\lambda_{n}^{c}\right)$ for various $n$ and $c$ (left), and graph of $\log _{10}\left(\lambda_{n}^{c}\right)$ (marked by "o") vs $\log _{10}\left(\nu_{n}^{c}\right)$ (marked by "+") for various $n$ and $c=20,30, \ldots, 90$ (right).

So far, intensive work has been devoted to developing efficient methods for computing the PSWFs and their eigenvalues (see, e.g., 44, 29, 8, 13, 16]). A package of Matlab programs for manipulating PSWFs is also available (cf. Boyd [8]). Following Bouwkamp [4] (also see, e.g., [33]), we expand $\psi_{n}^{c}(x)$ in terms of normalized Legendre polynomials: $\psi_{n}^{c}(x)=\sum_{k=0}^{\infty} \beta_{k}^{n} \widetilde{L}_{k}(x)$, and substitute the expansion into (2.1), which leads to the equivalent eigenproblem:

$$
\left(\mathbf{A}-\chi_{n}^{c} \cdot \mathbf{I}\right) \vec{\beta}^{n}=0, \quad n=0,1, \ldots,
$$


where $\vec{\beta}^{n}=\left(\beta_{0}^{n}, \beta_{1}^{n}, \beta_{2}^{n}, \ldots\right)^{t} \in l^{2}$ and $\mathbf{A}$ is a symmetric five-diagonal matrix with three non-zero diagonals given by

$$
\begin{aligned}
& A_{k, k}=k(k+1)+\frac{2 k(k+1)-1}{(2 k-1)(2 k+3)} \cdot c^{2}, \\
& A_{k, k+2}=A_{k+2, k}=\frac{(k+1)(k+2)}{(2 k+3) \sqrt{(2 k+1)(2 k+5)}} \cdot c^{2},
\end{aligned}
$$

for all $k=0,1,2, \ldots$ It is clear that the eigensystem (2.18) involves infinitely many unknowns, so a suitable truncation is needed. Boyd [8] suggested a safe and conservative cutoff number $M=2 N+30$, which guarantees a very high degree of accuracy (close to machine zero) calculation of all the first $N+1$ PSWFs $\left\{\psi_{n}^{c}\right\}_{n=0}^{N}$ and eigenvalues $\left\{\chi_{n}^{c}\right\}_{n=0}^{N}$ for all

$$
0 \leq c \leq c_{N}^{*}=\frac{\pi}{2}\left(N+\frac{1}{2}\right) .
$$

Here, $c_{N}^{*}$ is referred to as the "transition bandwidth" (the feasible $c$ should be in the range (2.20) to ensure the approximability (see [7])).

We now describe an algorithm for computing the eigenvalues $\left\{\lambda_{n}^{c}\right\}$ in (2.10). Taking $x=0$ in (2.10) leads to

$$
\mathrm{i}^{n} \lambda_{n}^{c} \psi_{n}^{c}(0)=\int_{-1}^{1} \psi_{n}^{c}(t) d t .
$$

The parity of the PSWFs and the fact that $\psi_{n}^{c}(x)$ has exactly $n$ real roots in $(-1,1)$ imply that $\psi_{n}^{c}(0) \neq 0$ for even $n$, while $\psi_{n}^{c}(0)=0$ for odd $n$. Therefore, we have

$$
\lambda_{n}^{c}=\frac{1}{\mathrm{i}^{n} \psi_{n}^{c}(0)} \int_{-1}^{1} \psi_{n}^{c}(t) d t=\frac{\sqrt{2} \beta_{0}^{n}}{\mathrm{i}^{n} \psi_{n}^{c}(0)}, \quad \text { for even } n,
$$

where we have used the fact that $\int_{-1}^{1} \psi_{n}^{c}(t) d t=\int_{-1}^{1} \sum_{k=0}^{\infty} \beta_{k}^{n} \widetilde{L}_{k}(t) d t=\sqrt{2} \beta_{0}^{n}$ in the last step. To calculate $\lambda_{n}^{c}$ with odd $n$, we differentiate (2.10) with respect to $x$ and then take $x=0$, which gives

$$
\lambda_{n}^{c}=\frac{c}{\mathrm{i}^{n-1} \partial_{x} \psi_{n}^{c}(0)} \int_{-1}^{1} t \psi_{n}^{c}(t) d t=\sqrt{\frac{2}{3}} \frac{c \beta_{1}^{n}}{\mathrm{i}^{n-1} \partial_{x} \psi_{n}^{c}(0)}, \quad \text { for odd } n .
$$

It should be pointed out that although the magnitude of $\lambda_{n}^{c}$ is exponentially small for large $n$, its evaluation through (2.21) -(2.22) is stable since the values of $\left|\psi_{n}^{c}(0)\right|$ (for even $n$ ) and $\left|\partial_{x} \psi_{n}^{c}(0)\right|$ (for odd $n$ ) are bounded below away from zero for all $n$.

Remark 1. As a consequence of (2.21), we have $\left|\beta_{0}^{n}\right|=\frac{1}{\sqrt{2}} \lambda_{n}^{c}\left|\psi_{n}^{c}(0)\right|$. Hence, for even $n \gg 1$, we have from (2.5) and (2.17) that

$$
\left|\beta_{0}^{n}\right| \sim \sqrt{n} \exp (\tilde{n}(\kappa-\log \tilde{n})), \quad \kappa=\log \frac{e c}{4}, \quad \tilde{n}=n+\frac{1}{2} .
$$

For odd $n$, we have from Stirling's formula that

$$
\left|\tilde{L}_{n}^{\prime}(0)\right|=\sqrt{\frac{2 n+1}{2}} \frac{(n+1) !}{2^{n}\left(\frac{n-1}{2}\right) !\left(\frac{n+1}{2}\right) !} \stackrel{\sqrt{2.15}}{\sim} n,
$$

and therefore, by Theorem 11 in $22,\left|\partial_{x} \psi_{n}^{c}(0)\right| \sim n$. Consequently, $\left|\beta_{1}^{n}\right|$ shares the same asymptotic behavior with $\left|\beta_{0}^{n}\right|$ as in (2.23). Notice that these estimates are more precise than those in Lemma A.2 of [10], which played an important role in the derivations of the main approximation result stated in Theorem 3.2. 


\section{Approximation by PSWFs}

A series of papers by Slepian et al. 25, 19, 26] and some recent works by Xiao and Rokhlin et al. [33, 32, 24, 22, have demonstrated that the PSWFs are an optimal tool for approximating bandlimited functions. Some error estimates were reported in 22, 32. Firstly, we will show that a super-geometric convergence can be achieved for a PSWF series approximation of bandlimited functions. However, our main concerns are with the approximation of general non-periodic functions in Sobolev spaces.

Define the finite-dimensional space

$$
X_{N}^{c}=\operatorname{span}\left\{\psi_{k}^{c}: 0 \leq k \leq N\right\}
$$

and denote by

$$
\left(\pi_{N}^{c} u\right)(x)=\sum_{k=0}^{N} \hat{u}_{k} \psi_{k}^{c}(x) \in X_{N}^{c} \quad \text { with } \quad \hat{u}_{k}=\int_{-1}^{1} u(x) \psi_{k}^{c}(x) d x,
$$

the $L^{2}$-orthogonal projection $\pi_{N}^{c}: L^{2}(I) \rightarrow X_{N}^{c}$ satisfying

$$
\left(\pi_{N}^{c} u-u, v_{N}\right)=0, \quad \forall v_{N} \in X_{N}^{c} .
$$

Theorem 3.1. Let $f(x)$ be a bandlimited function with a bandwidth $c>0$, defined by

$$
f(x)=\int_{-1}^{1} e^{i c x t} \phi(t) d t \quad \text { with } \quad \phi \in L^{2}(I)
$$

and let $f_{N}=\pi_{N}^{c} f=\sum_{k=0}^{N} \hat{f}_{k} \psi_{k}^{c}$. Then,

$$
\left\|f-f_{N}\right\| \leq \lambda_{N+1}^{c}\|\phi\| \lesssim \exp (\hat{N}(\kappa-\log \hat{N}))\|\phi\|,
$$

where $\kappa=\log \frac{e c}{4}$ and $\hat{N}=N+\frac{3}{2}$.

Proof. It is clear that by (2.10) and (3.4),

$$
\begin{aligned}
\hat{f}_{k} & =\int_{-1}^{1} f(x) \psi_{k}^{c}(x) d x \stackrel{\sqrt[3.4]{=}}{=} \int_{-1}^{1}\left[\int_{-1}^{1} e^{\mathrm{i} c x t} \phi(t) d t\right] \psi_{k}^{c}(x) d x \\
& =\int_{-1}^{1}\left[\int_{-1}^{1} e^{\mathrm{i} c x t} \psi_{k}^{c}(x) d x\right] \phi(t) d t=\mathrm{i}^{k} \lambda_{k}^{c} \int_{-1}^{1} \psi_{k}^{c}(t) \phi(t) d t \stackrel{2.10}{=} \mathrm{i}^{k} \lambda_{k}^{c} \hat{\phi}_{k},
\end{aligned}
$$

where $\hat{\phi}_{k}$ is the $(k+1)$ th coefficient in the PSWF expansion of $\phi$. Therefore, by the orthogonality condition (2.2), (2.11) and (3.6),

$$
\left\|f-f_{N}\right\|^{2}=\sum_{k=N+1}^{\infty} \hat{f}_{k}^{2}=\sum_{k=N+1}^{\infty}\left|\lambda_{k}^{c}\right|^{2} \hat{\phi}_{k}^{2} \leq\left|\lambda_{N+1}^{c}\right|^{2}\|\phi\|^{2} .
$$

Finally, the desired result follows from (2.17).

It is interesting to study the approximation of non-periodic functions by the PSWFs. The first result was derived in [10, 7] as stated below, whose proof was based on a delicate analysis of the decay property of the coefficients $\left\{\beta_{k}^{n}\right\}$ in (2.18).

Theorem 3.2. (Theorem 3.1 in [10]). Let $u \in H^{s}(I)$ with $s \geq 0$ and write

$$
u(x)=\sum_{k=0}^{\infty} \hat{u}_{k} \psi_{k}^{c}(x) \quad \text { with } \quad \hat{u}_{k}=\int_{-1}^{1} u(x) \psi_{k}^{c}(x) d x .
$$


Then, for

$$
q_{N}=\sqrt{\frac{c^{2}}{\chi_{N}^{c}}}<1,
$$

we have

$$
\left|\hat{u}_{N}\right| \leq C\left(N^{-\frac{2}{3} s}\|u\|_{H^{s}(I)}+\left(q_{N}\right)^{\delta N}\|u\|_{L^{2}(I)}\right),
$$

where $C$ and $\delta$ are positive constants independent of $u, N$ and $c$. Boyd [7] stated a similar result with

$$
\rho_{N}=\frac{c}{c_{N}^{*}}<1 \quad \text { with } \quad c_{N}^{*}=\frac{\pi}{2}\left(N+\frac{1}{2}\right),
$$

in place of $q_{N}$.

Remark 2. We see that if $c$ satisfies (3.9) or (3.11), the spectral accuracy can be achieved for sufficiently smooth $u$. In practice, we oftentimes choose $c=O(N)$ so as to obtain quasi-uniform computational grids. We also point out that the estimate (3.10) is suboptimal in terms of the order $O\left(N^{-\frac{2}{3} s}\right)$ (which is expected to be $O\left(N^{-s}\right)$ at least for a fixed bandwidth parameter $c$ ). As an example, we take $u(x)=e^{\mathrm{i} c x t}$, and derive from (2.10), (2.4) and (2.16) (also refer to Theorem 3.1) that

$$
\hat{u}_{N}=\mathrm{i}^{N} \lambda_{N}^{c} \psi_{N}^{c}(1) \quad \Rightarrow \quad\left|\hat{u}_{N}\right| \sim \exp (\tilde{N}(\kappa-\log \tilde{N}))
$$

with $\kappa=\log \frac{e c}{4}$ and $\tilde{N}=N+\frac{1}{2}$. Therefore, $\left|\hat{u}_{N}\right|$ decays super-geometrically, when $N>\frac{e}{4} c-\frac{1}{2}$. This behavior was also numerically confirmed by Figure 4 in Boyd [7. However, the estimate (3.10) only yields that $\left|\hat{u}_{N}\right| \leq C\left(\frac{c^{3 / 2}}{N}\right)^{\frac{2 s}{3}}$, so $\left|\hat{u}_{N}\right|$ decays exponentially when $N>c^{3 / 2}$. Hence, the estimate (3.10) is a little conservative.

In the sequel, we analyze the approximation properties of PSWFs using a different approach. For this purpose, we define the Sturm-Liouville operator associated with (2.1):

$$
\mathcal{D}_{c} u=-\partial_{x}\left(\left(1-x^{2}\right) \partial_{x} u\right)+c^{2} x^{2} u=-\left(1-x^{2}\right) u^{\prime \prime}(x)+2 x u^{\prime}(x)+c^{2} x^{2} u .
$$

One verifies readily that $\mathcal{D}_{c}$ is a compact, strictly positive and selfadjoint operator. Indeed, for any $u$ and $v$ in the domain of $\mathcal{D}_{c}$, applying integration by parts leads to

$$
\left(\mathcal{D}_{c} u, v\right)=\left(u, \mathcal{D}_{c} v\right)=a_{c}(u, v) ; \quad\left(\mathcal{D}_{c} u, u\right)=a_{c}(u, u)>0, \quad \text { if } u \neq 0,
$$

where the bilinear form

$$
a_{c}(\phi, \psi)=\left(\phi^{\prime}, \psi^{\prime}\right)_{\omega}+c^{2}(x \phi, x \psi) \text { with } \omega=1-x^{2} .
$$

Since $\mathcal{D}_{c} \psi_{n}^{c}=\chi_{n}^{c} \psi_{n}^{c}$, the following orthogonality follows from (3.14):

$$
a_{c}\left(\psi_{n}^{c}, \psi_{m}^{c}\right)=\left(\mathcal{D}_{c} \psi_{n}^{c}, \psi_{m}^{c}\right)=\chi_{n}^{c}\left(\psi_{n}^{c}, \psi_{m}^{c}\right)=\chi_{n}^{c} \delta_{m n} .
$$

To measure the truncation error $u-\pi_{N}^{c} u$, we introduce a Hilbert space associated with the Sturm-Liouville operator $\mathcal{D}_{c}$ in (3.13). Since $\mathcal{D}_{c}$ is a compact, symmetric and (strictly) positive selfadjoint operator, the fractional power $\mathcal{D}_{c}^{1 / 2}$ is well defined, and the associated norms can be characterized by (see, e.g., Chapter II of [28]):

$$
\left\|\mathcal{D}_{c}^{1 / 2} u\right\|^{2}=a_{c}(u, u) \quad \Rightarrow \quad\left\|\mathcal{D}_{c}^{m+1 / 2} u\right\|^{2}=a_{c}\left(\mathcal{D}_{c}^{m} u, \mathcal{D}_{c}^{m} u\right), \quad \forall m \in \mathbb{N} .
$$


For any integer $r \geq 0$, we introduce the Hilbert space:

$$
\widetilde{H}_{c}^{r}(I)=\left\{u \in L^{2}(I):\|u\|_{\widetilde{H}_{c}^{r}}^{2}=\left\|\mathcal{D}_{c}^{r / 2} u\right\|^{2}=\left(\mathcal{D}_{c}^{r / 2} u, \mathcal{D}_{c}^{r / 2} u\right)<\infty\right\},
$$

while for real $r \geq 0, \widetilde{H}_{c}^{r}(I)$ is defined by space interpolation as in [1]. Formally, we derive from the orthogonality (2.2) and (3.16) that for any $m \in \mathbb{N}$,

$$
\left\|\mathcal{D}_{c}^{m} u\right\|^{2}=\sum_{k=0}^{\infty}\left(\chi_{k}^{c}\right)^{2 m}\left|\hat{u}_{k}\right|^{2}, \quad\left\|\mathcal{D}_{c}^{m+1 / 2} u\right\|^{2}=a_{c}\left(\mathcal{D}_{c}^{m}, \mathcal{D}_{c}^{m}\right)=\sum_{k=0}^{\infty}\left(\chi_{k}^{c}\right)^{2 m+1}\left|\hat{u}_{k}\right|^{2} .
$$

Therefore, the norm of the space $\widetilde{H}_{c}^{r}(I)$ with real $r \geq 0$ can be characterized in the frequency space as

$$
\|u\|_{\widetilde{H}_{c}^{r}}=\left\|\mathcal{D}_{c}^{r / 2} u\right\|=\left(\sum_{k=0}^{\infty}\left(\chi_{k}^{c}\right)^{r}\left|\hat{u}_{k}\right|^{2}\right)^{1 / 2}, \quad c>0 .
$$

An equivalent norm expressed in terms of derivatives of $u$ with explicit dependence on $c$ will be presented later.

The fundamental approximation result is stated below.

Theorem 3.3. For any $u \in \widetilde{H}_{c}^{r}(I)$ with $r \geq 0$,

$$
\left\|\pi_{N}^{c} u-u\right\| \leq\left(\chi_{N+1}^{c}\right)^{-\frac{r}{2}}\|u\|_{\widetilde{H}_{c}^{r}} \leq N^{-r}\|u\|_{\widetilde{H}_{c}^{r}}
$$

in general, for $0 \leq \mu \leq r$,

$$
\left\|\pi_{N}^{c} u-u\right\|_{\widetilde{H}_{c}^{\mu}} \leq\left(\chi_{N+1}^{c}\right)^{-\frac{\mu-r}{2}}\left\|\pi_{N}^{c} u-u\right\|_{\widetilde{H}_{c}^{r}} \leq\left(\chi_{N+1}^{c}\right)^{-\frac{\mu-r}{2}}\|u\|_{\widetilde{H}_{c}^{r}} \leq N^{\mu-r}\|u\|_{\widetilde{H}_{c}^{r}} .
$$

Proof. We first assume that $r=2 m$. Since $\mathcal{D}_{c} \psi_{n}^{c}=\lambda_{n}^{c} \psi_{n}^{c}$, we derive from (3.14) that

$$
\begin{aligned}
\hat{u}_{k} & =\int_{-1}^{1} u(x) \psi_{k}^{c}(x) d x=\frac{1}{\left(\chi_{k}^{c}\right)^{m}} \int_{-1}^{1} u(x) \mathcal{D}_{c}^{m} \psi_{k}^{c}(x) d x \\
& \left.=\frac{1}{\left(\chi_{k}^{c}\right)^{m}} \int_{-1}^{1} \psi_{k}^{c}(x) \mathcal{D}_{c}^{m} u(x) d x=\frac{1}{\left(\chi_{k}^{c}\right)^{m}} \widehat{\left(\mathcal{D}_{c}^{m} u\right.}\right)_{k},
\end{aligned}
$$

where $\left.\widehat{\left(\mathcal{D}_{c}^{m} u\right.}\right)_{k}$ is the $(k+1)$ th coefficient of the prolate spheroidal expansion of $\mathcal{D}_{c}^{m} u$. Therefore, by (2.2), (2.3) and (3.23),

$$
\begin{array}{r}
\left.\left\|\pi_{N}^{c} u-u\right\|^{2}=\sum_{k=N+1}^{\infty}\left|\hat{u}_{k}\right|^{2}=\sum_{k=N+1}^{\infty}\left(\chi_{k}^{c}\right)^{-2 m} \mid \widehat{\left(\mathcal{D}_{c}^{m} u\right.}\right)\left._{k}\right|^{2} \\
\leq \max _{k>N}\left\{\left(\chi_{k}^{c}\right)^{-2 m}\right\}\left\|\mathcal{D}_{c}^{m} u\right\|^{2} \leq\left(\chi_{N+1}^{c}\right)^{-2 m}\|u\|_{\widetilde{H}_{c}^{2 m}}^{2},
\end{array}
$$

which, together with (2.6), yields (3.21) with $r=2 m$.

We now prove (3.21) with $r=2 m+1$. By (2.3) and (3.20),

$$
\begin{gathered}
\left\|\pi_{N}^{c} u-u\right\|^{2}=\sum_{k=N+1}^{\infty}\left|\hat{u}_{k}\right|^{2} \leq \max _{k>N}\left\{\left(\chi_{k}^{c}\right)^{-(2 m+1)}\right\} \sum_{k=N+1}^{\infty}\left(\chi_{k}^{c}\right)^{2 m+1}\left|\hat{u}_{k}\right|^{2} \\
\leq\left(\chi_{N+1}^{c}\right)^{-(2 m+1)} a_{c}\left(\mathcal{D}_{c}^{m} u, \mathcal{D}_{c}^{m} u\right)=\left(\chi_{N+1}^{c}\right)^{-(2 m+1)}\|u\|_{\widetilde{H}_{c}^{2 m+1}}^{2} .
\end{gathered}
$$

The above two estimates, together with a space interpolation, lead to (3.21). 
Next, by (2.3), (2.6) and (3.20), we have that for $0 \leq \mu \leq r$,

$$
\begin{aligned}
\| \pi_{N}^{c} u & -u \|_{\widetilde{H}_{c}^{\mu}}^{2}=\sum_{k=N+1}^{\infty}\left(\chi_{k}^{c}\right)^{\mu}\left|\hat{u}_{k}\right|^{2} \leq \max _{k>N}\left\{\left(\chi_{k}^{c}\right)^{\mu-r}\right\} \sum_{k=N+1}^{\infty}\left(\chi_{k}^{c}\right)^{r}\left|\hat{u}_{k}\right|^{2} \\
& =\left(\chi_{N+1}^{c}\right)^{\mu-r}\left\|\pi_{N}^{c} u-u\right\|_{\widetilde{H}_{c}^{r}}^{2} \leq\left(\chi_{N+1}^{c}\right)^{\mu-r} \sum_{k=0}^{\infty}\left(\chi_{k}^{c}\right)^{r}\left|\hat{u}_{k}\right|^{2} \\
& =\left(\chi_{N+1}^{c}\right)^{\mu-r}\|u\|_{\widetilde{H}_{c}^{r}}^{2} \leq N^{2(\mu-r)}\|u\|_{\widetilde{H}_{c}^{r}}^{2} .
\end{aligned}
$$

This completes the proof.

The norm in the upper bounds of the above estimates is expressed in the frequency space and implicitly depends on the bandwidth parameter $c$. To extract more explicit information from (3.21) and (3.22), it is necessary to express the norm in terms of derivatives of $u$ featured with an explicit dependence on $c$. Notice that setting $c=0$ in Theorem 3.3 we recover the Legendre polynomial approximation (see [14]), and we are able to show that

$$
\|u\|_{\widetilde{H}_{0}^{r}}^{2} \leq C \sum_{k=0}^{r} \int_{-1}^{1}\left|\partial_{x}^{k} u\right|^{2}\left(1-x^{2}\right)^{k} d x,
$$

where $C$ is a positive constant independent of $u$. A natural question is whether such a sharp upper bound, with the weight functions varying with the order of the derivatives and with explicitly depending on the bandwidth parameter $c$, is also available for $c>0$. That is,

$$
\|u\|_{\widetilde{H}_{c}^{r}}^{2} \leq C \sum_{k=0}^{r} \int_{-1}^{1} c^{2(r-k)}\left|\partial_{x}^{k} u\right|^{2}\left(1-x^{2}\right)^{k} d x .
$$

Indeed, one derives by using integration by parts that

$$
\begin{aligned}
\|u\|_{\widetilde{H}_{c}^{1}}^{2}= & a_{c}(u, u)=\int_{-1}^{1}\left|\partial_{x} u\right|^{2}\left(1-x^{2}\right) d x+c^{2} \int_{-1}^{1} x^{2} u^{2}(x) d x, \\
\|u\|_{\widetilde{H}_{c}^{2}}^{2}= & \left\|\mathcal{D}_{c} u\right\|^{2}=\int_{-1}^{1}\left|\partial_{x}^{2} u\right|^{2}\left(1-x^{2}\right)^{2} d x+2 \int_{-1}^{1}\left(1+c^{2} x^{2}\right)\left|\partial_{x} u\right|^{2}\left(1-x^{2}\right) d x \\
& +c^{2} \int_{-1}^{1}\left(c^{2} x^{4}+6 x^{2}-2\right)|u|^{2} d x, \\
\|u\|_{\widetilde{H}_{c}^{3}}^{2}= & a_{c}\left(\mathcal{D}_{c} u, \mathcal{D}_{c} u\right)=\int_{-1}^{1}\left|\partial_{x}^{3} u\right|^{2}\left(1-x^{2}\right)^{3} d x+\int_{-1}^{1}\left(8+3 c^{2} x^{2}\right)\left|\partial_{x}^{2} u\right|^{2}\left(1-x^{2}\right)^{2} d x \\
& +\int_{-1}^{1} P(x ; c)\left|\partial_{x} u\right|^{2}\left(1-x^{2}\right) d x+c^{2} \int_{-1}^{1} Q(x ; c)|u|^{2} d x,
\end{aligned}
$$

where

$P(x ; c)=3 c^{4} x^{4}+34 c^{2} x^{2}-8 c^{2}+4 ; \quad Q(x ; c)=c^{4} x^{6}+26 c^{2} x^{4}-14 c^{2} x^{2}+36 x^{2}-12$.

However, the verification process appears to be very tedious and lengthy for general $r>3$, although we believe (3.25) is still valid. 
We next present a considerably rough upper bound for the norm $\|\cdot\|_{\widetilde{H}_{c}^{r}}$. Indeed, by the definition (3.13) and an induction,

$$
\mathcal{D}_{c}^{m} u=\sum_{k=0}^{2 m}\left(1-x^{2}\right)^{(m-k)^{+}} c^{2[k / 2]} p_{j}\left(x ; c^{-2}\right) \partial_{x}^{2 m-k} u
$$

where $(m-k)^{+}=\max \{m-k, 0\},[a]$ denotes the maximum integer $\leq a$, and $p_{j}\left(x ; c^{-2}\right)$ are some generic polynomials of degree $\leq 2 m$ with coefficients involving $c^{-2 l}$ with $0 \leq l \leq m$. Without loss of generality, we assume that the bandwidth is bounded away from zero, i.e, $c \geq c_{0}>0$ for some positive constant $c_{0}$. Thus, by (3.18) and (3.27),

$$
\|u\|_{\widetilde{H}_{c}^{2 m}}=\left\|\mathcal{D}_{c}^{m} u\right\| \leq C \sum_{k=0}^{2 m} c^{2[k / 2]}\left\|\left(1-x^{2}\right)^{(m-k)^{+}} \partial_{x}^{2 m-k} u\right\|
$$

where $C$ is a positive constant independent of $c, u$ (but depends on $c_{0}$ ). We now consider the case with $r=2 m+1$. It is clear that

$$
\sqrt{1-x^{2}} \partial_{x} \mathcal{D}_{c}^{m} u=\sum_{k=0}^{2 m+1}\left(1-x^{2}\right)^{\frac{1}{2}+(m+1-k)^{+}} c^{2[(k-1) / 2]} q_{j}\left(x ; c^{-2}\right) \partial_{x}^{2 m+1-k} u
$$

where $\left\{q_{j}\left(x ; c^{-2}\right)\right\}$ are generic (uniformly bounded) polynomials as $\left\{p_{j}\left(x ; c^{-2}\right)\right\}$ as in (3.27). Hence, by the definitions (3.15) and (3.18),

$$
\begin{aligned}
& \|u\|_{\widetilde{H}_{c}^{2 m+1}}=\left(\int_{-1}^{1}\left|\partial_{x} \mathcal{D}_{c}^{m} u\right|^{2}\left(1-x^{2}\right) d x+c^{2} \int_{-1}^{1} x^{2}\left|\mathcal{D}_{c}^{m} u\right|^{2} d x\right)^{1 / 2} \\
& \leq\left\|\left(1-x^{2}\right)^{m+1 / 2} \partial_{x}^{2 m+1} u\right\|+C \sum_{k=1}^{2 m+1} c^{2[(k-1) / 2]+\frac{1}{2}}\left\|\left(1-x^{2}\right)^{(m+1-k)^{+}} \partial_{x}^{2 m+1-k} u\right\| .
\end{aligned}
$$

As a consequence of (3.28) and (3.30), the imbedding relation holds for the usual Sobolev space $H^{r}(I) \subseteq \widetilde{H}_{c}^{r}(I)$, and

$$
\|u\|_{\widetilde{H}_{c}^{r}} \leq C\left(1+c^{2}\right)^{r / 2}\|u\|_{H^{r}} .
$$

We derived above some upper bounds of the norm of the space in (3.18) and Theorem 3.3 characterized by the Sturm-Liouville operator. It is important to notice that the powers of the bandwidth parameter $c$ (and likewise for the weight function $\left.\left(1-x^{2}\right)^{k}\right)$ for derivatives of different orders are different (cf. (3.25)-(3.30) $)$, which turns out to be crucial for the convergence of PSWF expansions when we choose $c=O(N)$.

Before further establishing some estimates in higher-order Sobolev spaces, we first numerically illustrate that the estimate in Theorem 3.3 is optimal. For this purpose, we consider the approximation of a function with finite regularity by the truncated PSWF series. Consider

$$
u(x)=(x-a)^{\alpha} e^{\sin x} \text { with } a=0,1 \text {, and } \alpha=5 / 3 .
$$

It is clear that $u \in H^{r}(I)$ for any $r<\alpha+\frac{1}{2}$. Theorem 3.2 implies that if $c$ satisfies (3.9) or (3.11), then for $a=0$ or 1 ,

$$
\left|\hat{u}_{N+1}^{c}\right| \sim E_{N}^{c}:=\left\|\pi_{N}^{c} u-u\right\|=O\left(N^{\varepsilon-\frac{2}{3}\left(\alpha+\frac{1}{2}\right)}\right), \quad \forall \varepsilon>0 .
$$


We now examine the estimate in Theorem 3.3 A direct calculation shows that $\mathcal{D}_{c} u=u_{1}+c^{2} u_{2}$, where for $a=0$, we have $u_{1} \in H^{\alpha-\frac{3}{2}+\varepsilon}(I)$ and $u_{2} \in H^{\alpha+\frac{1}{2}+\varepsilon}(I)$ with $r<\alpha+\frac{1}{2}$. Therefore, as in the proof of Theorem 3.3.

$$
\begin{aligned}
\hat{u}_{N+1}^{c}= & \int_{-1}^{1} u \psi_{N+1}^{c} d x=\frac{1}{\chi_{N+1}^{c}} \int_{-1}^{1} \mathcal{D}_{c} u \psi_{N+1}^{c} d x=\frac{1}{\chi_{N+1}^{c}} \int_{-1}^{1} u_{1} \psi_{N+1}^{c} d x \\
& +\frac{c^{2}}{\left(\chi_{N+1}^{c}\right)^{2}} \int_{-1}^{1} \mathcal{D}_{c} u_{2} \psi_{N+1}^{c} d x .
\end{aligned}
$$

We see that the error is dominated by the highest derivative term (where $c$ is not involved) even when $c^{2}=O\left(\chi_{N+1}^{c}\right)$. This process can be continued if $u_{2}$ enjoys more regularity. Based on this observation, we conclude from Theorem 3.3 that for any $\varepsilon>0$,

$$
\left|\hat{u}_{N+1}^{c}\right| \sim E_{N}^{c}=\left\|\pi_{N}^{c} u-u\right\|= \begin{cases}O\left(N^{\varepsilon-\left(\alpha+\frac{1}{2}\right)}\right), & \text { if } a=0 \\ O\left(N^{\varepsilon-(2 \alpha+1)}\right), & \text { if } a=1\end{cases}
$$
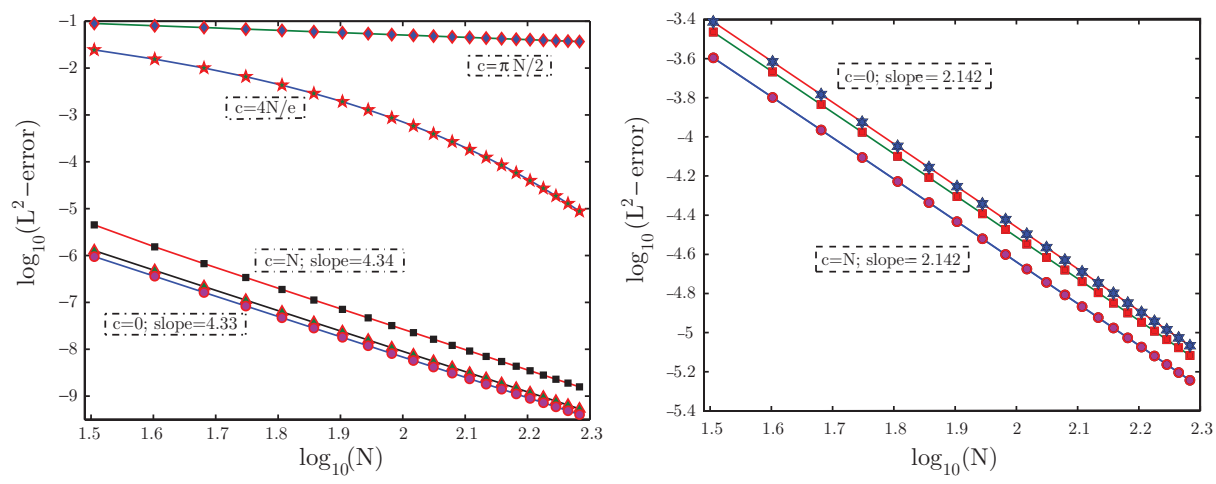

Figure 3. The errors $E_{N}^{c}$ of the PSWF approximation of (3.32) in the log-log scale. Left: $a=1$; Right: $a=0$.

We notice that the order of convergence roughly should be the slope of the error $E_{N}^{c}$ against $N$ in the $\log$-log scale. In Figure 3 (left), we plot the $L^{2}$-error $E_{N}^{c}$ in the $\log$ - $\log$ scale for $a=1, N \in[32,196]$ and $c=0, N / 2, N, 4 N / e, \pi N / 2$. We also calculate the slope (modulo the minus sign) of the error lines for $c=0, N / 2, N$, which approximately equals 4.33 and agrees with the predicted convergence order $2 \alpha+1=\frac{13}{4} \approx 4.333$ in (3.35). We visualize from Figure 3 (left) that the PSWFs with smaller $c$ ( $c=0$ corresponds to the Legendre polynomials) give better approximations than those with large $c$, since the singularity of the derivative appears near the endpoint $x=1$, and the points are denser near $x= \pm 1$ for small $c$. We also notice that as $c$ stretches its limit: $c=4 N / e, \frac{\pi}{2} N$ (cf. (2.17) and (3.11)), the accuracy deteriorates significantly.

In Figure 3 (right), we depict the errors for the PSWF approximation of (3.32) with $a=0$ for $c=0, N / 2, N$. It indicates a convergence rate $O\left(N^{-2.142}\right)$ consistent with the prediction in (3.35) (note: $\alpha+\frac{1}{2}=\frac{13}{6} \approx-2.167$ ). It is worthwhile to point out that the PSWF approximation with $c=N, N / 2$ produces a better accuracy than that with smaller $c$. This is mainly because the PSWFs oscillate 
more uniformly as $c$ increases, which thereby improves the resolution near the center $x=0$

We are now in a position to estimate the truncation error: $u-\pi_{N}^{c} u$ in higherorder Sobolev spaces.

Theorem 3.4. For any $v \in \widetilde{H}_{c}^{r}(I)$,

$$
\left\|\partial_{x}\left(\pi_{N}^{c} u-u\right)\right\|_{\omega} \leq\left(1+c N^{-1}\right) N^{1-r}\|u\|_{\widetilde{H}_{c}^{r}}, \quad r \geq 1,
$$

and

$$
\left\|\partial_{x}^{2}\left(\pi_{N}^{c} u-u\right)\right\|_{\omega^{2}} \lesssim\left(1+c N^{-1}+c^{2} N^{-2}\right) N^{2-r}\|u\|_{\widetilde{H}_{c}^{r}}, \quad r \geq 2,
$$

where the weight functions $\omega=1-x^{2}$ and $\omega^{2}=\left(1-x^{2}\right)^{2}$.

Proof. By the first identity of (3.26) and Theorem 3.3.

$$
\left\|\partial_{x}\left(\pi_{N}^{c} u-u\right)\right\|_{\omega} \leq\left\|\pi_{N}^{c} u-u\right\|_{\widetilde{H}_{c}^{1}}+c\left\|\pi_{N}^{c} u-u\right\| \leq\left(1+c N^{-1}\right) N^{1-r}\|u\|_{\widetilde{H}_{c}^{r}} .
$$

This gives (3.36).

Similarly, by the second identity of (3.26),

$$
\begin{aligned}
\left\|\partial_{x}^{2}\left(\pi_{N}^{c} u-u\right)\right\|_{\omega^{2}} \leq & \left\|\pi_{N}^{c} u-u\right\|_{\widetilde{H}_{c}^{2}}+\sqrt{2\left(1+c^{2}\right)}\left\|\partial_{x}\left(\pi_{N}^{c} u-u\right)\right\|_{\omega} \\
& +c \sqrt{4+c^{2}}\left\|\pi_{N}^{c} u-u\right\| .
\end{aligned}
$$

Thus, the result (3.37) follows from Theorem 3.3 and (3.36).

Remark 3. The estimate $\left\|\partial_{x}^{k}\left(\pi_{N}^{c} u-u\right)\right\|_{\omega^{k}}=O\left(N^{k-r}\right)$ for $r \geq k \geq 3$ can be derived in a similar fashion.

As a consequence of Theorem 3.4 we have the following suboptimal estimate in the $H^{1}$-norm.

Corollary 3.1. For any $u \in \widetilde{H}_{c}^{r}(I)$ with $r \geq 2$,

$$
\left\|\partial_{x}\left(\pi_{N}^{c} u-u\right)\right\| \lesssim\left(1+c N^{-1}+c^{2} N^{-2}\right) N^{2-r}\|u\|_{\widetilde{H}_{c}^{r}} .
$$

Proof. We first recall an imbedding inequality (see Formula (13.5) in 2]):

$$
\left\|\partial_{x} u\right\| \leq 2\left(\left\|\partial_{x}^{2} u\right\|_{\omega^{2}}+\left\|\partial_{x} u\right\|_{\omega^{2}}\right) \leq 2\left(\left\|\partial_{x}^{2} u\right\|_{\omega^{2}}+\left\|\partial_{x} u\right\|_{\omega}\right),
$$

which, together with Theorem 3.4, implies the desired result.

We now estimate the truncation error $u-\pi_{N}^{c} u$ in the $L^{\infty}$-norm.

Theorem 3.5. For any $u \in \widetilde{H}_{c}^{r}(I)$ with $r>1$,

$$
\left\|\pi_{N}^{c} u-u\right\|_{L^{\infty}} \leq \sqrt{\frac{2}{r-1}} N^{1-r}\|u\|_{\widetilde{H}_{c}^{r}} .
$$

Proof. By (2.5), the Cauchy-Schwarz inequality and (3.20),

$$
\begin{aligned}
\left|\left(\pi_{N}^{c} u-u\right)(x)\right| & \leq \sum_{k=N+1}^{\infty}\left|\hat{u}_{k}\right| \max _{|x| \leq 1}\left|\psi_{k}^{c}(x)\right| \leq \sum_{k=N+1}^{\infty} k\left|\hat{u}_{k}\right| \\
& \leq 2\left(\sum_{k=N+1}^{\infty} k\left(\chi_{k}^{c}\right)^{-r}\right)^{\frac{1}{2}}\left(\sum_{k=N+1}^{\infty}\left(\chi_{k}^{c}\right)^{r}\left|\hat{u}_{k}^{c}\right|^{2}\right)^{\frac{1}{2}} \\
& \leq 2\left(\sum_{k=N+1}^{\infty} k\left(\chi_{k}^{c}\right)^{-r}\right)^{\frac{1}{2}}\|u\|_{\widetilde{H}_{c}^{r}} .
\end{aligned}
$$


Since $\left(\chi_{k}^{c}\right)^{-1}<k^{-2}$ (cf. (2.6) $)$, we obtain that

$$
\sum_{k=N+1}^{\infty} k\left(\chi_{k}^{c}\right)^{-r} \leq \sum_{k=N+1}^{\infty} k^{1-2 r} \leq \int_{N+1}^{\infty} x^{1-2 r} d x \leq \frac{1}{2(r-1)} N^{2-2 r} .
$$

Plugging this into (3.41) yields the desired result.

As the conclusion of this section, we derive two inverse inequalities, which are useful for the analysis of non-linear problems.

Theorem 3.6. For any $\phi \in X_{N}^{c}$,

$$
\|\phi\|_{\widetilde{H}_{c}^{r}} \leq\left(\chi_{N}^{c}\right)^{\frac{r}{2}}\|\phi\| \leq(N+c+1)^{r}\|\phi\|, \quad \forall r \geq 0 ;
$$

in particular, we have

$$
\begin{aligned}
& \left\|\partial_{x} \phi\right\|_{\omega} \leq(N+2 c+1)\|\phi\|, \\
& \left\|\partial_{x}^{2} \phi\right\|_{\omega^{2}} \leq\left(N^{2}+(3 c+1) N+3(c+1)^{2}\right)\|\phi\|,
\end{aligned}
$$

where the weight functions $\omega=1-x^{2}$ and $\omega^{2}=\left(1-x^{2}\right)^{2}$.

Proof. For any $\phi \in X_{N}^{c}$, we write

$$
\phi(x)=\sum_{k=0}^{N} \hat{\phi}_{k} \psi_{k}^{c}(x) \quad \text { with } \quad \hat{\phi}_{k}=\int_{-1}^{1} \phi(x) \psi_{k}^{c}(x) d x .
$$

It is clear that by (3.19), (2.3) and Lemma 2.2

$$
\|\phi\|_{\widetilde{H}_{c}^{r}}^{2}=\sum_{k=0}^{N}\left(\chi_{k}^{c}\right)^{r}\left|\hat{\phi}_{k}\right|^{2} \leq\left(\chi_{N}^{c}\right)^{r}\|\phi\|^{2} \leq\left(N(N+1)+c^{2}\right)^{r}\|\phi\|^{2},
$$

which implies (3.42).

Next, by the first identity of (3.26) and (3.42) with $r=1$,

$$
\left\|\partial_{x} \phi\right\|_{\omega} \leq\|\phi\|_{\widetilde{H}_{c}^{1}}+c\|\phi\| \leq(N+2 c+1)\|\phi\| .
$$

Similarly, by the second identity of (3.42), (3.26) and (3.44),

$$
\left\|\partial_{x}^{2} \phi\right\|_{\omega^{2}} \lesssim\|\phi\|_{\widetilde{H}_{c}^{2}}+c\left\|\partial_{x} \phi\right\|_{\omega}+c^{2}\|\phi\| \leq\left(N^{2}+(3 c+1) N+3(c+1)^{2}\right)\|\phi\| .
$$

This ends the proof.

In summary, we have presented a set of PSWF approximation results in Sobolev spaces, which not only demonstrate the feasibility of using the PSWFs to approximate non-periodic functions on finite intervals, but also serve as a fundamental tool for the analysis of PSWF spectral methods for PDEs.

\section{PSWF SPECTRAL METHODS}

As the PSWFs continue to enjoy applications in signal processing, wave scattering and a variety of engineering fields, there has been a growing recent interest in developing methods using the PSWFs as basis functions, which include in particular the PSWF collocation/pseudospectral methods and spectral-element methods. The main advantages of the PSWF approach over the polynomial-based counterpart are addressed in [7, 10, 3, 17. In this section, we first briefly introduce the PSWF pseudospectral method, and then propose and analyze a PSWF spectral-Galerkin scheme for elliptic-type PDEs. We also provide some illustrative numerical results to support our analysis. 
4.1. PSWF quadrature and interpolation. In practice, two types of quadrature rules are used. The first one is a direct extension of the LegendreGauss-Lobatto rule, where the quadrature points $\left\{\xi_{N, j}^{c}\right\}_{j=0}^{N}$ are the roots of $\left(1-x^{2}\right) \partial_{x} \psi_{N}^{c}(x)$, and the corresponding weights $\left\{\rho_{N, j}^{c}\right\}_{j=0}^{N}$ are determined by

$$
\int_{-1}^{1} \psi_{n}^{c}(x) d x=\sum_{j=0}^{N} \psi_{n}^{c}\left(\xi_{N, j}^{c}\right) \rho_{N, j}^{c}, \quad n=0,1, \ldots, N .
$$

The second rule uses the quadrature points and weights $\left\{\zeta_{N, j}^{c}, \sigma_{N, j}^{c}\right\}_{n=0}^{N}$ (with $\zeta_{N, 0}^{c}=-1$ and $\zeta_{N, N}^{c}=1$ ), determined by

$$
\int_{-1}^{1} \psi_{n}^{c}(x) d x=\sum_{j=0}^{N} \psi_{n}^{c}\left(\zeta_{N, j}^{c}\right) \sigma_{N, j}^{c}, \quad n=0,1, \ldots, 2 N-1 .
$$

The Matlab programs for computing the points and weights associated with these two formulas can be found in [8]. For convenience, we denote by $\left\{x_{j}^{c}, \omega_{j}^{c}\right\}_{j=0}^{N}$ one of the above sets of points and weights. Let $X_{N}^{c}$ be the finite-dimensional space defined in (3.1). The Lagrange-like PSWF basis $\left\{h_{j}^{c}\right\}_{j=0}^{N}$ is defined by

$$
h_{j}^{c}(x)=\sum_{k=0}^{N} \alpha_{k}^{j} \psi_{k}^{c}(x) \in X_{N}^{c}, \quad j=0,1, \ldots, N,
$$

where the coefficients $\left\{\alpha_{k}^{j}\right\}$ are uniquely determined by $h_{j}^{c}\left(x_{i}^{c}\right)=\delta_{i j}, 0 \leq i, j \leq N$. Hence, for any $u \in X_{N}^{c}$, we have that

$$
u(x)=\sum_{j=0}^{N} u\left(x_{j}\right) h_{j}^{c}(x) .
$$

Equipped with the nodal basis and the associated quadrature rule, the PSWF collocation and pseudospectral methods can be built up and implemented essentially in the same fashion as the Legendre or Chebyshev methods (see, e.g., 9, 15]).

4.2. PSWF spectral-Galerkin methods. Next, we propose a viable PSWF spectral-Galerkin method. To fix the main idea, we focus on the analysis of a one-dimensional model equation, and we present some numerical results for a twodimensional problem later on.

Now consider

$$
-u^{\prime \prime}(x)+\gamma u(x)=f(x), \quad \text { in } I=(-1,1), \quad u( \pm 1)=0,
$$

where $\gamma \geq 0$ and $f$ is a given function. We now introduce a modal basis for $H_{0}^{1}(I):=\left\{u \in H^{1}(I): u( \pm 1)=0\right\}$ using compact combinations of integration of PSWFs. Define

$$
\Phi_{j}^{c}(x)=a_{j} \Psi_{j}^{c}(x)+b_{j} \Psi_{j+2}^{c}(x), \quad j \geq 0,
$$

where

$$
\Psi_{j}^{c}(x)=\int_{-1}^{x} \psi_{j}^{c}(t) d t, \quad j \geq 0
$$


and the coefficients $\left\{a_{j}, b_{j}\right\}$ are chosen such that $a_{j}=1$ and $b_{j}=0$ for odd $j$, and for even $j$,

$$
a_{j}=\frac{\Psi_{j+2}^{c}(1)}{\sqrt{\left[\Psi_{j}^{c}(1)\right]^{2}+\left[\Psi_{j+2}^{c}(1)\right]^{2}}}, \quad b_{j}=-\frac{\Psi_{j}^{c}(1)}{\sqrt{\left[\Psi_{j}^{c}(1)\right]^{2}+\left[\Psi_{j+2}^{c}(1)\right]^{2}}} .
$$

Since $\Psi_{j}^{c}(-1)=0$ for all $j$ and $\Psi_{j}^{c}(1)=0$ for odd $j$, we have $\Phi_{j}^{c}( \pm 1)=0$ for all $j \geq 0$.

Remark 4. This basis can be viewed as an extension of the compact combinations of the Legendre polynomials used in, e.g., [23].

Define the finite-dimensional approximation space

$$
Y_{N}^{c}=\operatorname{span}\left\{\Phi_{0}^{c}, \Phi_{1}^{c}, \ldots, \Phi_{N-2}^{c}\right\} .
$$

The PSWF spectral-Galerkin approximation of (4.5) is to find $u_{N} \in Y_{N}^{c}$ such that

$$
\left(u_{N}^{\prime}, v_{N}^{\prime}\right)+\gamma\left(u_{N}, v_{N}\right)=\left(f, v_{N}\right), \quad \forall v_{N} \in Y_{N}^{c} .
$$

One verifies readily from the Lax-Milgram lemma that this problem admits a unique solution $u_{N} \in Y_{N}^{c}$ if $f \in L^{2}(I)$.

4.2.1. Error analysis. Using a standard argument for the analysis of Galerkin methods leads to

$$
\left\|u-u_{N}\right\|_{1} \leq \inf _{v_{N} \in Y_{N}^{c}}\left\|u-v_{N}\right\|_{1} .
$$

Hence, to analyze the error, we have to study the approximation property of the modal basis defined in (4.6). For any $u \in H_{0}^{1}(I)$, we write

$$
u(x)=\sum_{n=0}^{\infty} \tilde{u}_{n} \Phi_{n}^{c}(x)=\sum_{n=0}^{\infty}\left(a_{n} \tilde{u}_{n}+b_{n-2} \tilde{u}_{n-2}\right) \Psi_{n}^{c}(x),
$$

where we assume that $\tilde{u}_{-2}=\tilde{u}_{-1}=0$. Since $\left|a_{n}\right|,\left|b_{n}\right| \leq 1$, we have from (3.20) that for $r \geq 1$,

$$
\begin{aligned}
\left\|\partial_{x} u\right\|_{\widetilde{H}_{c}^{r-1}} & =\left(\sum_{n=0}^{\infty}\left(\chi_{n}^{c}\right)^{r-1}\left|a_{n} \tilde{u}_{n}+b_{n-2} \tilde{u}_{n-2}\right|^{2}\right)^{\frac{1}{2}} \\
& \leq\left(\sum_{n=0}^{\infty}\left(\chi_{n}^{c}\right)^{r-1}\left(\left|\tilde{u}_{n}\right|^{2}+\left|\tilde{u}_{n-2}\right|^{2}\right)\right)^{\frac{1}{2}} \leq \sqrt{2}\left(\sum_{n=0}^{\infty}\left(\chi_{n}^{c}\right)^{r-1}\left|\tilde{u}_{n}\right|^{2}\right)^{\frac{1}{2}} .
\end{aligned}
$$

For $r \geq 1$, we define the space

$\widehat{H}_{c}^{r}(I):=\left\{u \in H_{0}^{1}(I): \partial_{x} u \in \widetilde{H}_{c}^{r-1}(I)\right.$ and $\left.\|u\|_{\widehat{H}_{c}^{r}}=\left(\sum_{n=0}^{\infty}\left(\chi_{n}^{c}\right)^{r-1}\left|\tilde{u}_{n}\right|^{2}\right)^{\frac{1}{2}}<\infty\right\}$.

Let $\left(\pi_{N}^{1} u\right)(x)=\sum_{n=0}^{N-2} \tilde{u}_{n} \Phi_{n}^{c}(x)$. The truncation error is characterized by the following theorem.

Theorem 4.1. For any $u \in \widehat{H}_{c}^{r}(I)$ with $r \geq 1$, we have

$$
\left\|\partial_{x}\left(u-\pi_{N}^{1} u\right)\right\| \leq \sqrt{2}\left(\chi_{N-1}^{c}\right)^{(1-r) / 2}\|u\|_{\widehat{H}_{c}^{r}} \lesssim N^{1-r}\|u\|_{\widehat{H}_{c}^{r}} .
$$


Proof. It is clear that

$$
\begin{aligned}
\partial_{x}\left(u-\pi_{N}^{1} u\right)= & \sum_{n=N-1}^{\infty} \tilde{u}_{n} \Phi_{n}^{c}=a_{N-1} \tilde{u}_{N-1} \psi_{N-1}^{c}+a_{N} \tilde{u}_{N} \psi_{N}^{c} \\
& +\sum_{n=N+1}^{\infty}\left(a_{n} \tilde{u}_{n}+b_{n-2} \tilde{u}_{n-2}\right) \psi_{n}^{c} .
\end{aligned}
$$

Hence, by the orthogonality (2.2) and the fact $\left|a_{n}\right|,\left|b_{n}\right| \leq 1$,

$$
\begin{aligned}
& \left\|\partial_{x}\left(u-\pi_{N}^{1} u\right)\right\|^{2}=\left|a_{N-1} \tilde{u}_{N-1}\right|^{2}+\left|a_{N} \tilde{u}_{N}\right|^{2}+\sum_{n=N+1}^{\infty}\left|a_{n} \tilde{u}_{n}+b_{n-2} \tilde{u}_{n-2}\right|^{2} \\
& \leq 2 \sum_{n=N-1}^{\infty}\left|\tilde{u}_{n}\right|^{2} \stackrel{2.31}{\leq} 2\left(\chi_{N-1}^{c}\right)^{r-1} \sum_{n=N-1}^{\infty}\left(\chi_{n}^{c}\right)^{1-r}\left|\tilde{u}_{n}\right|^{2} \stackrel{4.130}{\leq} 2\left(\chi_{N-1}^{c}\right)^{1-r}\|u\|_{\widehat{H}_{c}^{r}}^{2} \\
& \text { (2.6) } \\
& \lesssim N^{2(1-r)}\|u\|_{\widehat{H}_{c}^{r}}^{2} \text {. }
\end{aligned}
$$

This ends the proof.

As a consequence of (4.10) and Theorem 4.1, we obtain the convergence of the PSWF spectral-Galerkin approximation.

Corollary 4.1. Let $u$ and $u_{N}$ be the solutions of (4.5) and (4.9), respectively. If $u \in \widehat{H}_{c}^{r}(I)$ with $r \geq 1$, then

$$
\left\|u-u_{N}\right\|_{1} \lesssim N^{1-r}\|u\|_{\widehat{H}_{c}^{r}} .
$$

Proof. Using the Poincaré inequality:

$$
\|u\| \lesssim\left\|\partial_{x} u\right\|, \quad \forall u \in H_{0}^{1}(I)
$$

we derive from (4.10) and Theorem 4.1 that

$$
\left\|u-u_{N}\right\|_{1} \leq\left\|\pi_{N}^{1} u-u\right\|_{1} \lesssim\left\|\partial_{x}\left(\pi_{N}^{1} u-u\right)\right\| \lesssim N^{1-r}\|u\|_{\widehat{H}_{c}^{r}} .
$$

This completes the proof.

4.2.2. Numerical results. We next briefly describe the implementation of the proposed PSWF spectral-Galerkin method and present some illustrative numerical results.

Let us first examine the matrix of the system (4.9) under the basis (4.6). By (2.2) and (4.6),

$$
s_{i j}^{c}=\left(\partial_{x} \Phi_{j}^{c}, \partial_{x} \Phi_{i}^{c}\right)= \begin{cases}1 & \text { if } j=i, \\ a_{j} b_{i} & \text { if } j=i+2, \\ a_{i} b_{j} & \text { if } j=i-2, \\ 0 & \text { otherwise }\end{cases}
$$

We see that the stiffness matrix $\mathbf{S}_{c}=\left(s_{i j}\right)$ is symmetric and pentadiagonal with three non-zero diagonals, while the mass matrix $\mathbf{M}_{c}=\left(m_{i j}^{c}\right)$ with $m_{i j}^{c}=\left(\Phi_{j}^{c}, \Phi_{i}^{c}\right)$ is a full matrix with half zero entries: $m_{i j}=0$ if $i+j$ is odd. Let $\mathbf{u}_{c}=\left(\tilde{u}_{0}, \ldots, \tilde{u}_{N-2}\right)^{t}$ and $\mathbf{f}_{c}=\left(f_{0}, \ldots, \tilde{f}_{N-2}\right)^{t}$ with $f_{i}=\left(f, \Phi_{i}^{c}\right)$. Then the matrix form of (4.9) is

$$
\left(\mathbf{S}_{c}+\gamma \mathbf{M}_{c}\right) \mathbf{u}_{c}=\mathbf{f}_{c} .
$$


In the following test, we consider (4.5) with $\gamma=1$ and the exact solution: $u(x)=\sin (20 \pi x)$. Since the solution is infinitely smooth, the theoretical analysis predicts that if $c<N$, the error is expected to decay exponentially. We plot in Figure 4 the $\log _{10}\left(L^{2}\right.$-error) against $N \in[8,96]$ for various $c=0,20 \pi, N / 2,2 N / 3, N$, $4 N / e, \pi N / 2$. It indicates that the PSWF approximation with $c=20 \pi$ provides the best result (cf. Theorem 3.1), and a more rapid convergence is observed for $c=\alpha N$ as $\alpha$ increases from 0 to 1 . However, the accuracy deteriorates for $c>N$. We also see that the PSWFs with a suitable bandwidth parameter $c$ produce a better result than the Legendre approximation.

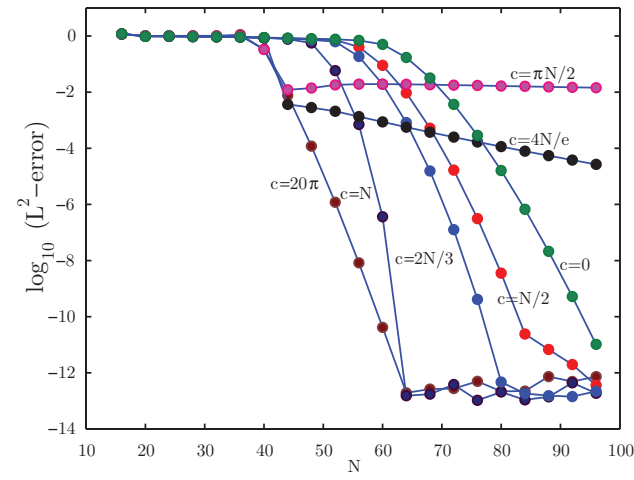

Figure $4 . \log _{10}\left(L^{2}\right.$-error) against $N$ for various $c=0,20 \pi, N / 2$, $2 N / 3, N, 4 N / e, \pi N / 2$.

We now apply the PSWF spectral-Galerkin method to the Poisson equation

$$
-\Delta u=f, \quad \text { in } \Omega=(-1,1)^{2},\left.\quad u\right|_{\partial \Omega}=0,
$$

and seek the numerical solution $u_{N}(x, y)=\sum_{k=0}^{N-2} \sum_{l=0}^{N-2} \tilde{u}_{k l} \Phi_{k}(x) \Phi_{l}(y)$ such that

$$
\left(\nabla u_{N}, \nabla v_{N}\right)=\left(f, v_{N}\right), \quad \forall v_{N} \in Y_{N}^{c} \times Y_{N}^{c}
$$

It is equivalent to solving the system

$$
\mathbf{S}_{c} \mathbf{U M}_{c}+\mathbf{M}_{c} \mathbf{U} \mathbf{S}_{c}=\mathbf{F}_{c}
$$

where the matrices $\mathbf{U}=\left(\tilde{u}_{k l}\right)$ and $\mathbf{F}_{c}=\left(f_{i j}^{c}\right)$ with $f_{i j}^{c}=\left(f, \Phi_{i}^{c} \Phi_{j}^{c}\right)$. We test the scheme with an exact solution $u(x, y)=\sin (4 \pi x) \sin (3 \pi y) \exp (x y)$. In Figure 5 (left), we plot the numerical solution $u_{48}(x, y)$ versus the exact solution $u(x, y)$, which are indistinguishable. We depict in Figure 5 (right) the $\log _{10}$ of the maximum pointwise errors (marked by circles) and $L^{2}$-errors (marked by squares) against $N$ for $c=0, N / 2$ and $N$. We observe a convergence behavior similar to the onedimensional case. In general, $c=N / 2$ gives a good approximation, which was also suggested by [10] for the PSWF pseudospectral method.

\section{CONCluding REMARKS}

The spheroidal wave functions of order zero have been proven to be an optimal tool for approximating bandlimited functions. In the mean time, as a generalization of the classical Legendre polynomials, the PSWF grids, with a suitable choice of the intrinsic bandwidth parameter $c=O(N)$, are more uniformly spaced than the Legendre points. As a result, the PSWF collocation/pseudospectral methods enjoy some remarkable advantages over the polynomial-based counterparts. Although 

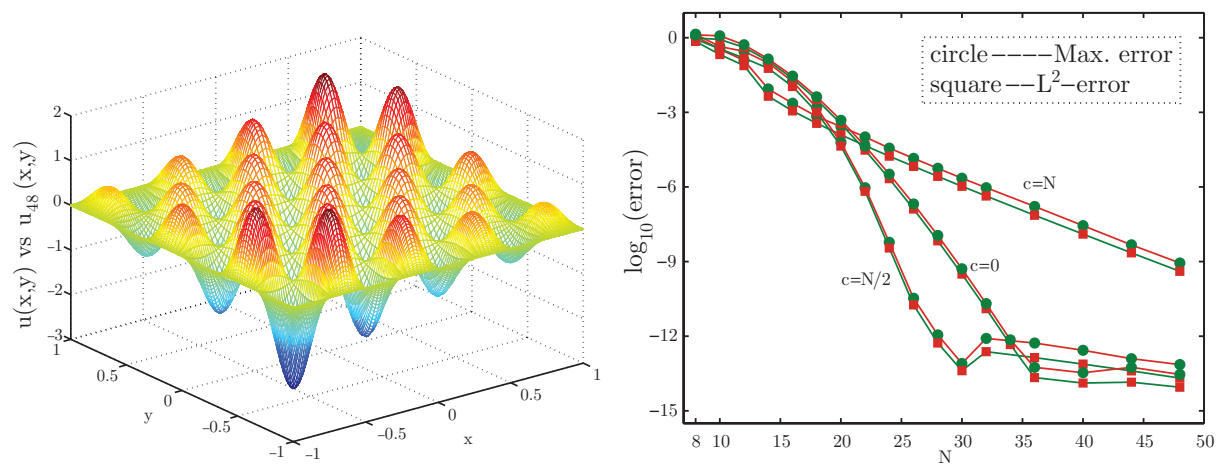

Figure 5. Numerical solution $u_{48}$ versus the exact solution $u$ (left), and $\log _{10}$ of the maximum pointwise errors (marked by circles) and $L^{2}$-errors (marked by squares) against $N$ for $c=0, N / 2$ and $N$.

there is a growing interest in developing PSWF-based methods, their approximation properties have only been studied to a limited degree. We presented in this paper a set of optimal approximation results on the PSWF expansions of nonperiodic functions in Sobolev spaces with norms characterized by the associated Sturm-Liouville operator. We also derived some upper bounds for these norms featured with an explicit dependence on $c$. These results are very similar to those for the Legendre expansions, and they are basic ingredients for the analysis of PSWF approximations to PDEs. We proposed a PSWF spectral-Galerkin method using a modal basis consisting of compact linear combinations of integration of the PSWFs, which provides a viable alternative to the PSWF pseudospectral/collocation methods. We also presented some illustrative numerical results and provided some useful guidelines for the choice of the bandwidth parameter.

\section{Appendix A. Proof of Lemma 2.2}

Differentiating the equation (2.1) with respect to $c$ yields

$$
\partial_{x}\left(\left(1-x^{2}\right) \partial_{x} \partial_{c} \psi_{n}^{c}\right)+\left(\chi_{n}^{c}-c^{2} x^{2}\right) \partial_{c} \psi_{n}^{c}=\left(2 c x^{2}-\partial_{c} \chi_{n}^{c}\right) \psi_{n}^{c} .
$$

Multiplying the above equation by $\psi_{n}^{c}$, and integrating the resulting equation over $(-1,1)$, we derive from the orthogonality (2.2) and integration by parts that

$$
\begin{aligned}
2 c \int_{-1}^{1} x^{2}\left[\psi_{n}^{c}(x)\right]^{2} d x-\frac{\partial \chi_{n}^{c}}{\partial c} & =\int_{-1}^{1}\left[\partial_{x}\left(\left(1-x^{2}\right) \partial_{x} \partial_{c} \psi_{n}^{c}\right)+\left(\chi_{n}^{c}-c^{2} x^{2}\right) \partial_{c} \psi_{n}^{c}\right] \psi_{n}^{c} d x \\
& =\int_{-1}^{1} \partial_{c} \psi_{n}^{c}\left[\partial_{x}\left(\left(1-x^{2}\right) \partial_{x} \psi_{n}^{c}\right)+\left(\chi_{n}^{c}-c^{2} x^{2}\right) \psi_{n}^{c}\right] d x \\
& =0 .
\end{aligned}
$$

Thus,

$$
0<\frac{\partial \chi_{n}^{c}}{\partial c}=2 c \int_{-1}^{1} x^{2}\left[\psi_{n}^{c}(x)\right]^{2} d x<2 c \quad \Rightarrow \quad 0<\chi_{n}^{c}-\chi_{n}^{0}<c^{2} .
$$

Since $\chi_{n}^{0}=n(n+1)$, the desired result follows. 


\section{ACKNOWLEDGMENT}

The author would like to thank Professor Huiyuan Li at the Institute of Software of Chinese Academy of Sciences for some thoughtful discussions.

\section{REFERENCES}

[1] R. A. Adams. Sobolov Spaces. Academic Press, New York, 1975. MR0450957 (56:9247)

[2] C. Bernardi and Y. Maday. Spectral method. In P. G. Ciarlet and L. L. Lions, editors, Handbook of Numerical Analysis, V. 5 (Part 2). North-Holland, 1997. MR1470226

[3] G. Beylkin and K. Sandberg. Wave propagation using bases for bandlimited functions. Wave Motion, 41(3):263-291, 2005. MR 2120171(2005i:76097)

[4] C. J. Bouwkamp. On the theory of spheroidal wave functions of order zero. Nederl. Akad. Wetensch., Proc., 53:931-944 = Indagationes Math. 12, 326-339 (1950), 1950. MR0036366 $(12: 97 \mathrm{f})$

[5] John P. Boyd. Approximation of an analytic function on a finite real interval by a bandlimited function and conjectures on properties of prolate spheroidal functions. Appl. Comput. Harmon. Anal., 15(2):168-176, 2003. MR2007058 (2004g:41027)

[6] John P. Boyd. Large mode number eigenvalues of the prolate spheroidal differential equation. Appl. Math. Comput., 145(2-3):881-886, 2003. MR2009306 (2004h:34169)

[7] John P. Boyd. Prolate spheroidal wavefunctions as an alternative to Chebyshev and Legendre polynomials for spectral element and pseudospectral algorithms. J. Comput. Phys., 199(2):688-716, 2004. MR2091911 (2005m:65288)

[8] John P. Boyd. Algorithm 840: Computation of grid points, quadrature weights and derivatives for spectral element methods using prolate spheroidal wave functions - prolate elements. $A C M$ Trans. Math. Software, 31(1):149-165, 2005. MR2291545

[9] C. Canuto, M. Y. Hussaini, A. Quarteroni, and T. A. Zang. Spectral methods. Scientific Computation. Springer-Verlag, Berlin, 2006. Fundamentals in single domains. MR2223552 (2007c:65001)

[10] Q.-Y. Chen, D. Gottlieb, and J. S. Hesthaven. Spectral methods based on prolate spheroidal wave functions for hyperbolic PDEs. SIAM J. Numer. Anal., 43(5):1912-1933 (electronic), 2005. MR2192324(2006k:65275)

[11] Dorota Dąbrowska. Recovering signals from inner products involving prolate spheroidals in the presence of jitter. Math. Comp., 74(249):279-290 (electronic), 2005. MR2085411 (2006d:94020)

[12] C. Flammer. Spheroidal Wave Functions. Stanford University Press, Stanford, CA, 1957. MR0089520 (19:689a)

[13] Andreas Glaser, Xiangtao Liu, and Vladimir Rokhlin. A fast algorithm for the calculation of the roots of special functions. SIAM J. Sci. Comput., 29(4):1420-1438 (electronic), 2007. MR2341794 (2009c:33056)

[14] Ben-yu Guo and Li-Lian Wang. Jacobi approximations in non-uniformly Jacobi-weighted Sobolev spaces. J. Approx. Theory, 128(1):1-41, 2004. MR.2063010 (2005h:41010)

[15] J. Hesthaven, S. Gottlieb, and D. Gottlieb. Spectral Methods for Time-Dependent Problems. Cambridge Monographs on Applied and Computational Mathematics. Cambridge, 2007.

[16] Abderrazek Karoui and Tahar Moumni. New efficient methods of computing the prolate spheriodal wave functions and their corresponding eigenvalues. Appl. Comput. Harmon. Anal., 24:269-289, 2008. MR2407005 (2009b:33032)

[17] N. Kovvali, W. Lin, and L. Carin. Pseudospectral method based on prolate spheroidal wave functions for frequency-domain electromagnetic simulations. IEEE Trans. Antennas and Propagation, 53:3990-4000, 2005.

[18] Narayan Kovvali, Wenbin Lin, Zhiqin Zhao, Luise Couchman, and Lawrence Carin. Rapid prolate pseudospectral differentiation and interpolation with the fast multipole method. SIAM J. Sci. Comput., 28(2):485-497 (electronic), 2006. MR2231717 (2007c:65085)

[19] H. J. Landau and H. O. Pollak. Prolate spheroidal wave functions, Fourier analysis and uncertainty. III. The dimension of the space of essentially time- and band-limited signals. Bell System Tech. J., 41:1295-1336, 1962. MR0147686 (26:5200) 
[20] J. Meixner and F. W. Schäfke. Mathieusche Funktionen und Sphäroidfunktionen mit Anwendungen auf physikalische und technische Probleme. Springer, Berlin, 1954. MR0066500 (16:586g)

[21] Ian C. Moore and Michael Cada. Prolate spheroidal wave functions, an introduction to the Slepian series and its properties. Appl. Comput. Harmon. Anal., 16(3):208-230, 2004. MR2054280 (2005d:33031)

[22] Vladimir Rokhlin and Hong Xiao. Approximate formulae for certain prolate spheroidal wave functions valid for large values of both order and band-limit. Appl. Comput. Harmon. Anal., 22(1):105-123, 2007. MR2287387 (2008a:33024)

[23] Jie Shen. Efficient spectral-Galerkin method I. Direct solvers for second- and fourthorder equations using Legendre polynomials. SIAM J. Sci. Comput., 15:1489-1505, 1994. MR.1298626 (95j:65150)

[24] Yoel Shkolnisky, Mark Tygert, and Vladimir Rokhlin. Approximation of bandlimited functions. Appl. Comput. Harmon. Anal., 21(3):413-420, 2006. MR2274847 (2008c:41023)

[25] D. Slepian and H. O. Pollak. Prolate spheroidal wave functions, Fourier analysis and uncertainty. I. Bell System Tech. J., 40:43-63, 1961. MR0140732 (25:4146)

[26] David Slepian. Prolate spheroidal wave functions, Fourier analysis and uncertainity. IV. Extensions to many dimensions; generalized prolate spheroidal functions. Bell System Tech. J., 43:3009-3057, 1964. MR0181766 (31:5993)

[27] Mark A. Taylor and Beth A. Wingate. A generalization of prolate spheroidal functions with more uniform resolution to the triangle. J. Engrg. Math., 56(3):221-235, 2006. MR2292673 (2008a:65044)

[28] Roger Temam. Infinite-dimensional dynamical systems in mechanics and physics, volume 68 of Applied Mathematical Sciences. Springer-Verlag, New York, 1988. MR953967 (89m:58056)

[29] G. Walter and T. Soleski. A new friendly method of computing prolate spheroidal wave functions and wavelets. Appl. Comput. Harmon. Anal., 19(3):432-443, 2005. MR2186452 (2006j:65053)

[30] Gilbert G. Walter. Prolate spheroidal wavelets: Translation, convolution, and differentiation made easy. J. Fourier Anal. Appl., 11(1):73-84, 2005. MR2128945 (2006a:42062)

[31] Gilbert G. Walter and Xiaoping Shen. Wavelets based on prolate spheroidal wave functions. J. Fourier Anal. Appl., 10(1):1-26, 2004. MR2045522 (2005c:42041)

[32] H. Xiao. Prolate spheroidal wave functions, quadrature, interpolation, and asymptotic formulae. Ph.D. Thesis, Yale University, 2001.

[33] H. Xiao, V. Rokhlin, and N. Yarvin. Prolate spheroidal wavefunctions, quadrature and interpolation. Inverse Problems, 17(4):805-838, 2001. Special issue to celebrate Pierre Sabatier's 65th birthday (Montpellier, 2000). MR.1861483 (2002h:41049)

[34] Ahmed I. Zayed. A generalization of the prolate spheroidal wave functions. Proc. Amer. Math. Soc., 135(7):2193-2203 (electronic), 2007. MR2299497(2008f:33013)

Division of Mathematical Sciences, School of Physical and Mathematical Sciences, Nanyang Technological University, 637371, Singapore

E-mail address: lilian@ntu.edu.sg 